\title{
Ring-shaped confocal photoacoustic computed tomography for small- animal whole-body imaging
}

Jun Xia, Muhammad Chatni, Konstantin Maslov, Zijian Guo, Mark Anastasio, et al.

Jun Xia, Muhammad Chatni, Konstantin Maslov, Zijian Guo, Mark Anastasio, Lihong V. Wang, "Ring-shaped confocal photoacoustic computed tomography for small-animal whole-body imaging," Proc. SPIE 8223, Photons Plus Ultrasound: Imaging and Sensing 2012, 82230G (23 February 2012); doi: $10.1117 / 12.906056$

SPIE. Event: SPIE BiOS, 2012, San Francisco, California, United States 


\title{
Ring-shaped confocal photoacoustic computed tomography for small- animal whole-body imaging
}

\author{
Jun Xia ${ }^{\mathrm{a}}$, Muhammad Chatni ${ }^{\mathrm{a}}$, Konstantin Maslov ${ }^{\mathrm{a}}, \mathrm{Zijian} \mathrm{Guo}^{\mathrm{a}}$, Mark Anastasio ${ }^{\mathrm{a}}$, and Lihong V. \\ Wang ${ }^{2 *}$. \\ ${ }^{a}$ Dept. of Biomedical Engineering, Washington University in St. Louis, St. Louis, MO USA 63130 \\ *Correspondence should be addressed to LHWang@biomed.wustl.edu
}

\begin{abstract}
We report herein a novel three-dimensional photoacoustic computed tomography (PACT) system for small-animal whole-body imaging. The PACT system, based on a 512-element full-ring ultrasonic transducer array, was cylindrically focused and capable of forming a two-dimensional image in 1.6 seconds. The pulsed laser could either illuminate directly from the top or be reshaped to illuminate the sample from the side. Top illumination was mainly used for mouse brain and mouse embryo imaging. Side illumination provided in vivo anatomical images of an adult mouse. By translating the mouse along the elevational direction, the system provided serial cross-sectional images.
\end{abstract}

Keywords: Photoacoustic computed tomography, small-animal imaging, whole-body imaging, focal-line image reconstruction

\section{INTRODUCTION}

Due to the widespread use of animal models for human disease studies, small-animal imaging plays an increasingly important role in biomedical research. Previously, the majority of small-animal whole-body imaging systems were based on magnetic resonance imaging (MRI) or X-ray computed tomography (X-ray CT) for anatomic evaluation, and positron emission tomography (PET) for functional evaluation. However, these imaging techniques have their own limitations, for instance, X-ray CT and PET utilize ionizing radiation, which may be carcinogenic and will confound experimental results in oncology, and MRI requires a very high magnetic field and long imaging time (more than one hour) for smallanimal imaging.

Recently, photoacoustic imaging emerged as a promising tool for small-animal imaging [1,2]. By combining optical sensitivity and ultrasonic imaging depth scalability, this hybrid technology provides high-resolution images beyond the diffusion limit of optical microscopic imaging technologies. Over the past few years, several array-based photoacoustic whole-body imaging systems have been proposed, including 64-element arc array [2,3], 64-element half-ring array [4], and 128-element hemisphere array [5]. However, these systems have either limited boundary coverage or a long data acquisition time, which limits their applications on pre-clinical research. In this report, we present a novel whole-body imaging system based on a 512-element full-ring ultrasonic transducer array.

\section{SYSTEM DESIGN}

Figure 1 shows the schematic diagram of the system. The key components include a 512-element full-ring ultrasonic transducer array, a 64-channel data acquisition (DAQ) system, and two laser systems.

The 512-element full-ring transducer array (Imasonic, Inc.) has a central frequency of $5 \mathrm{MHz}$ and a reception bandwidth greater than $80 \%$. The piezocomposite elements were spaced with a lateral pitch of one wavelength $(0.3 \mathrm{~mm})$ and a kerf of $0.1 \mathrm{~mm}$. The array element was mechanically shaped into an arc to produce an axial focal depth of $19 \mathrm{~mm}$ within the imaging plane. The combined foci of all elements generate a central imaging region of $20 \mathrm{~mm}$ diameter and 1 $\mathrm{mm}$ thickness. The elevation focus and the small pitch enable each array element to be treated as a point detector in a two-dimensional (2D) image reconstruction. During the experiment, signals acquired from all 512 elements were first amplified and selected by a custom-designed connector board consisting of 512 preamplifiers, 64 8:1 multiplexers, and

Photons Plus Ultrasound: Imaging and Sensing 2012, edited by Alexander A. Oraevsky, Lihong V. Wang, Proc. of SPIE Vol. 8223, 82230G · (c) 2012 SPIE · CCC code: 1605-7422/12/\$18 · doi: 10.1117/12.906056 
64 post-amplifiers, and then digitalized by a 64-channel data acquisition system [6]. The two stage amplification achieves a total gain of $60 \mathrm{~dB}$. Due to the 8:1 multiplexing, the current system can acquire one frame of data per 1.6 seconds.

The excitation source consists of two lasers. An optical parametric oscillator (OPO) laser, tunable from 400 to 680 $\mathrm{nm}$ and 740 to $2000 \mathrm{~nm}$, and a Ti:sapphire laser, tunable from 680 to $900 \mathrm{~nm}$. The laser beam was first homogenized using a ground glass, and then passed through a conical lens to form a ring-shaped light. The ring-shaped light was then focused using an optical condenser made from acrylic to project a light band around the animal. The thickness of the light band was $5 \mathrm{~mm}$, and the diameter was determined by the cross-sectional diameter of the animal. By removing the conical lens and the optical condenser, the system can also provide planar top illumination for brain [7] and embryo imaging [8].

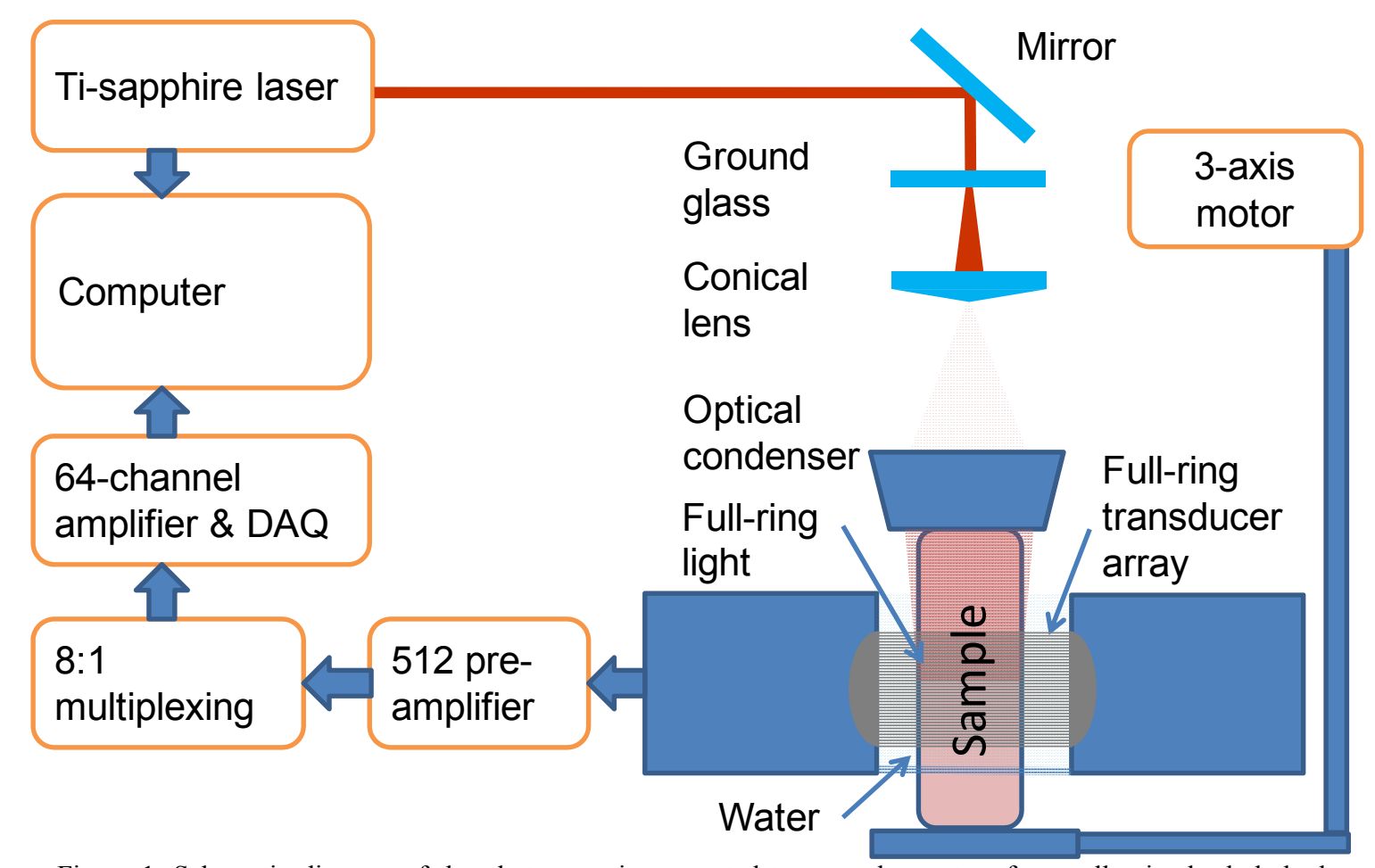

Figure 1. Schematic diagram of the photoacoustic computed tomography system for small-animal whole-body imaging.

\section{IMAGE RECONSTRUCTION}

Due the elevation focus of the transducer element, the detector cannot be treated as a point detector in a threedimensional (3D) image reconstruction. Instead, we can treat the focal line of the detector as an auxiliary line for applying appropriate delays in the 3D image reconstruction [8]. As shown in figure 2, to compute the delay of a point $\mathrm{A}$ in the 3D space, we first project A to the $x-y$ plane (B). Point B is then connected to the center point of the transducer $(\mathrm{C})$, crossing the focal line at point $\mathrm{D}$. We then connect $\mathrm{A}$ and $\mathrm{D}$, and extend the line to reach the transducer at $\mathrm{E}$. The line $\mathrm{AE}$ is always perpendicular to the transducer surface $(\mathrm{CD}=\mathrm{DE})$, and is used for calculating the delay time between the imaging point $\mathrm{A}$ and the transducer. In the $3 \mathrm{D}$ image reconstruction, the original time-domain photoacoustic signals from each transducer are back-projected into the 3D imaging space based on the calculated delay time, and are then summed to form the reconstructed image $[8,9]$. 


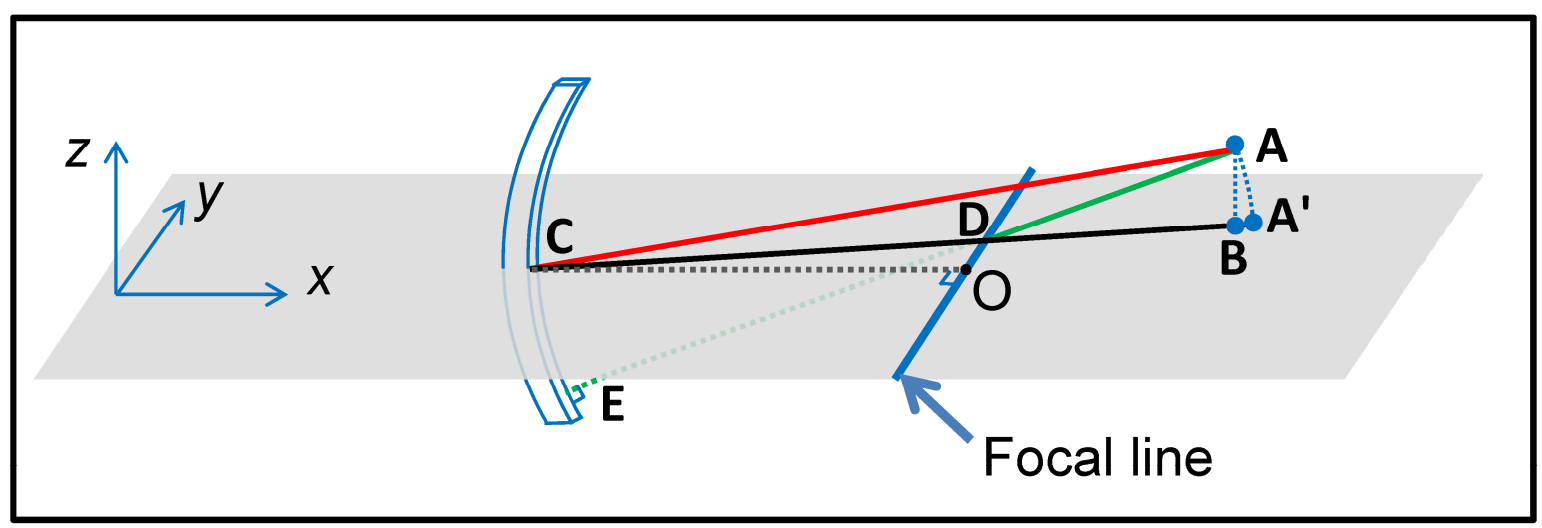

Figure 2. Schematic of the 2D reconstruction, 3D direct reconstruction, and 3D focal-line reconstruction concepts. A: point of reconstruction. $\mathrm{A}^{\prime}$ : point $\mathrm{A}$ projected in $2 \mathrm{D}$ reconstruction. $\mathrm{A}^{\prime} \mathrm{C}$ : $2 \mathrm{D}$ reconstruction delay. $\mathrm{AC}$ : $3 \mathrm{D}$ direction reconstruction delay. AE: 3D focal-line reconstruction delay. $x-y$ : $2 \mathrm{D}$ reconstruction plane. The focal line is perpendicular to the transducer plane $(x-z)$.

\section{RESUTLS}

Figure $3 \mathrm{a}$ is an in vivo cortical vasculature image of an adult mouse with intact skin and skull. Figure $3 \mathrm{~b}$ is an ex vivo image of a 15-day-old mouse embryo fixed in a gelatin gel. Figures $3 \mathrm{a}$ and $3 \mathrm{~b}$ were acquired over a depth of $6 \mathrm{~mm}$ and 9 $\mathrm{mm}$, respectively, and maximum-amplitude-projected along the elevation direction. The 3D focal-line reconstruction algorithm was utilized to generate these images. Figure $3 \mathrm{c}$ is an in vivo cross sectional image of an adult mouse. Figures $3 \mathrm{a}$ and $3 \mathrm{~b}$ were acquired using the top illumination scheme, and figure $3 \mathrm{c}$ was acquired using the side illumination scheme.

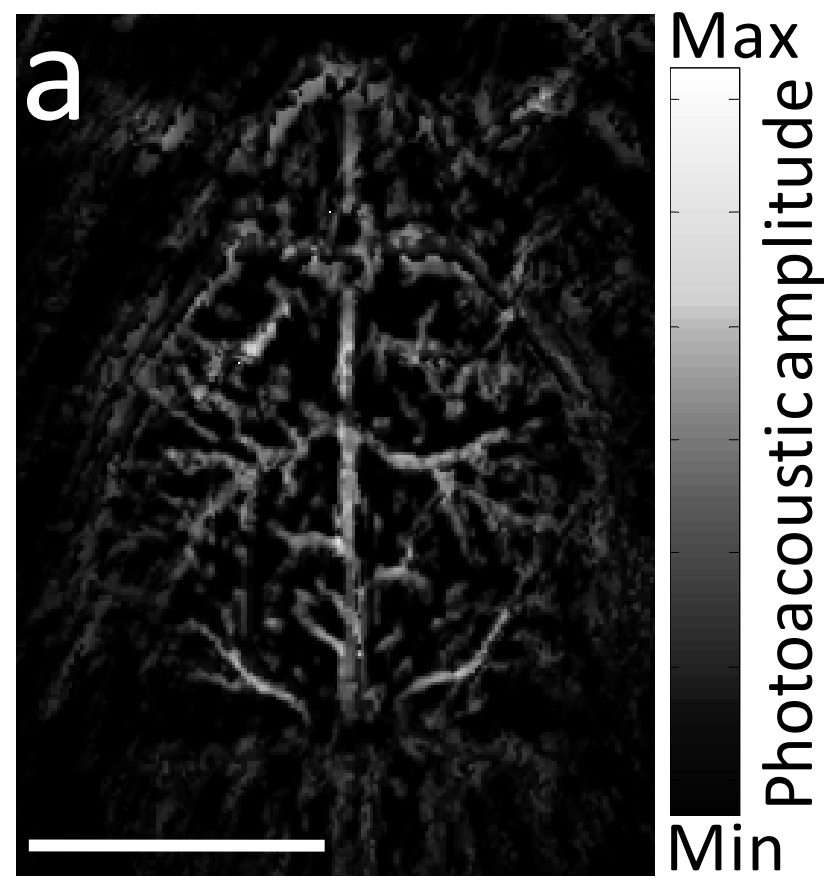




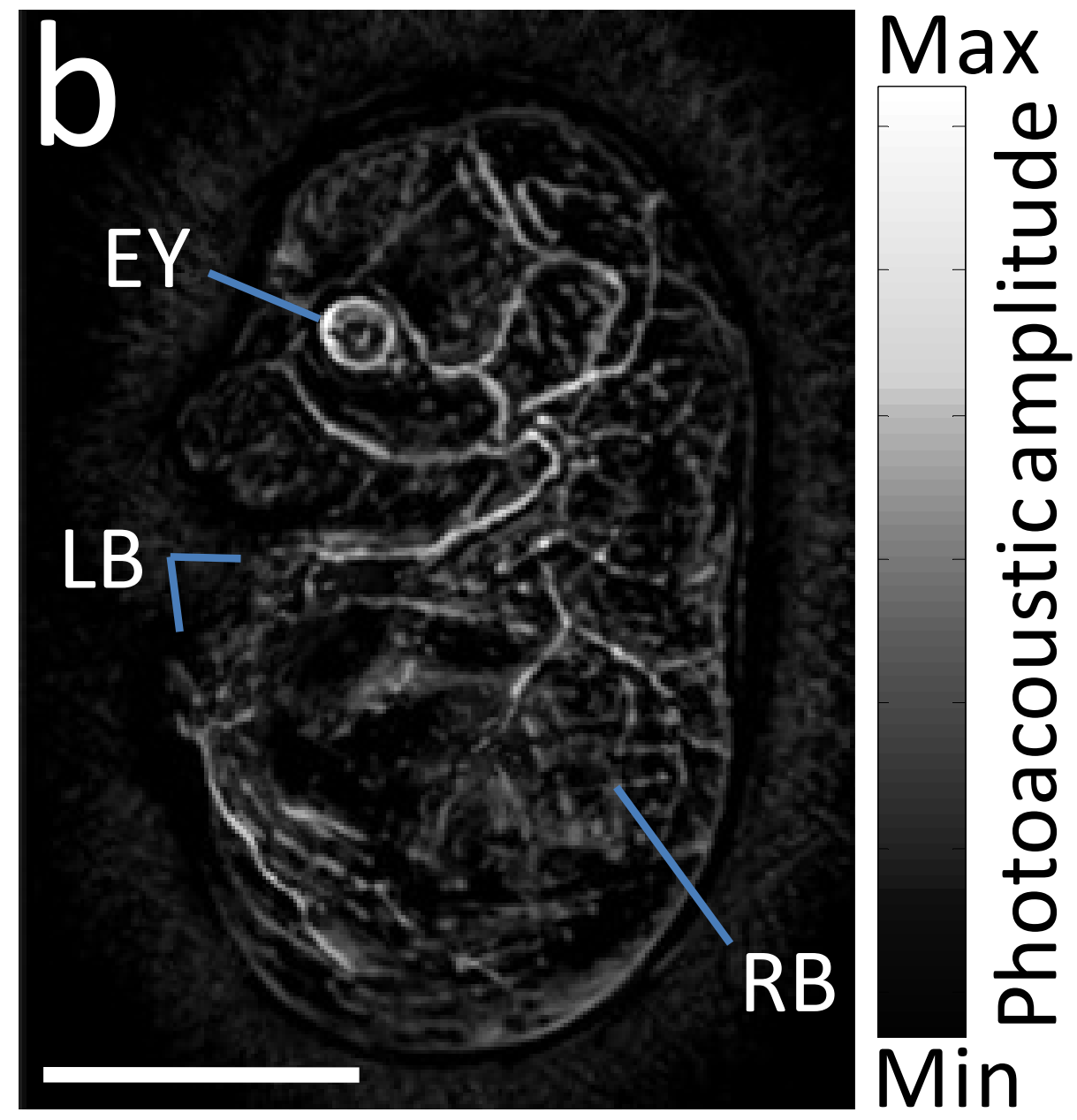

Proc. of SPIE Vol. 8223 82230G-4 


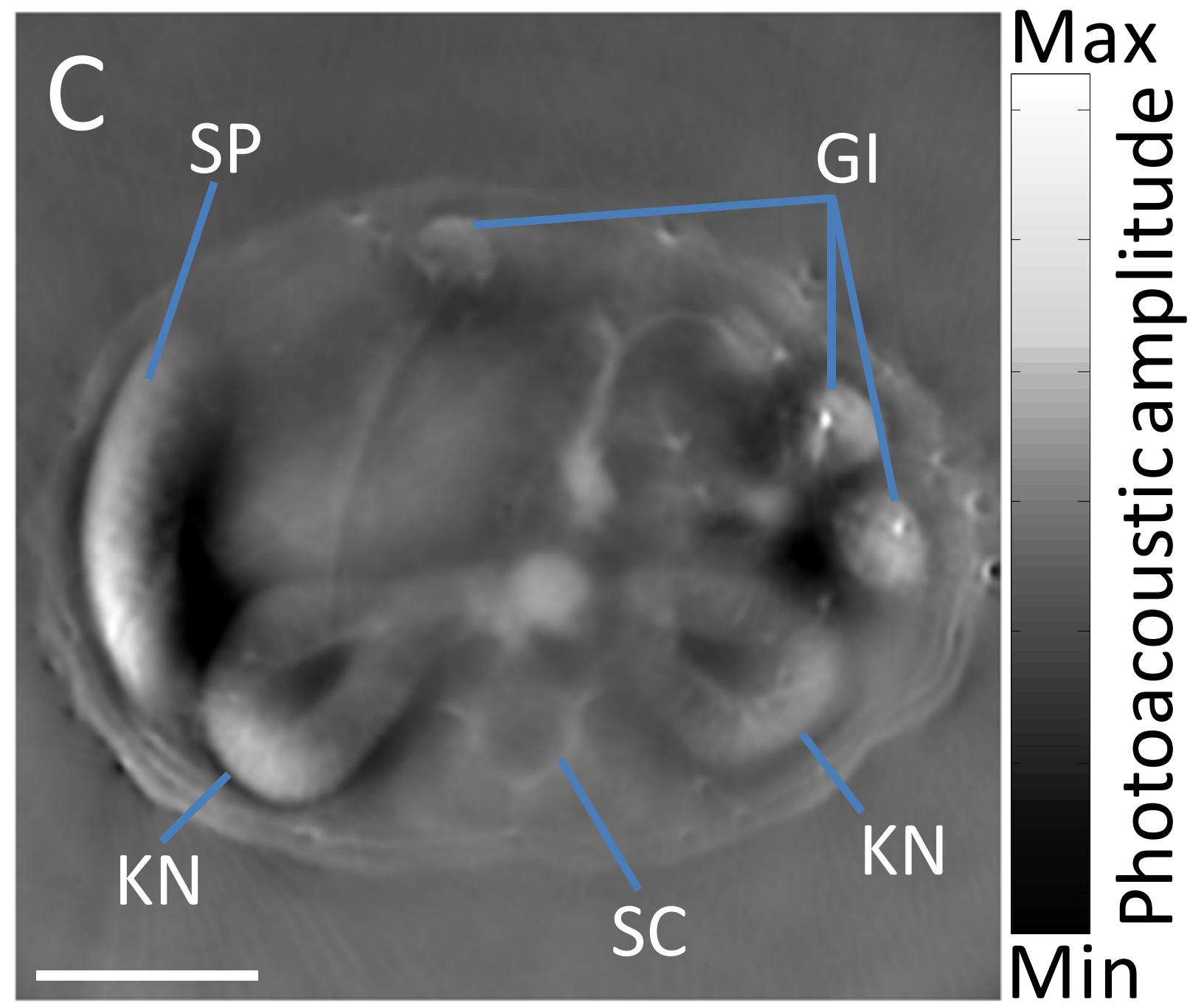

Figure 3. In vivo and ex vivo photoacoustic computed tomography images of mice. a. Maximum-amplitudeprojection image of the cortical vasculature in a living adult mouse. b. Maximum-amplitude-projection image of a 15-day-old mouse embryo fixed in a gelatin gel. c. Cross-sectional image of a living adult mouse. EY, eye; GI, GI tract; KN, kidney; LB, limbs; RB, ribs; SC, spinal cord; SP, spleen. Scale bar, 5 mm.

\section{CONCLUSION}

A new small-animal whole-body imaging system has been developed based on the full-ring ultrasonic transducer array. The new system has an in-plane resolution of $0.1 \mathrm{~mm}$ and a frame rate of one frame per 1.6 seconds. Images of the mouse brain, embryo, and kidneys were successfully acquired using the system. 


\section{ACKNOWLEDGEMENTS}

This work was sponsored in part by National Institutes of Health grants R01 EB000712, R01 EB008085, R01 CA134539, U54 CA136398, R01 EB010049, R01 CA157277, R01 CA159959, and 5P60 DK02057933. L.W. has financial interests in Microphotoacoustics, Inc. and Endra, Inc., which, however, did not support this work.

\section{REFERENCES}

[1] L. V. Wang, "Multiscale photoacoustic microscopy and computed tomography," Nature Photonics, 3(9), 503-509 (2009).

[2] H.-P. Brecht, R. Su, M. Fronheiser et al., "Whole-body three-dimensional optoacoustic tomography system for small animals," Journal of Biomedical Optics, 14(6), 064007-8 (2009).

[3] D. R. Reinecke, R. A. Kruger, R. B. Lam et al., "Co-registered photoacoustic, thermoacoustic, and ultrasound mouse imaging," Proceedings of SPIE, 7564, 756420-9 (2010).

[4] A. Buehler, E. Herzog, D. Razansky et al., "Video rate optoacoustic tomography of mouse kidney perfusion," Opt. Lett., 35(14), 2475-2477 (2010).

[5] R. Kruger, D. Reinecke, G. Kruger et al., "HYPR-spectral photoacoustic CT for preclinical imaging," Proceedings of SPIE, 7177, 71770F (2009).

[6] J. Gamelin, D. Castillo, Q. Zhu et al., [Design and Characterization of an Array-Based Photoacoustic Tomographic System for Small Animal Imaging] CRC Press, (2009).

[7] C. Li, A. Aguirre, J. Gamelin et al., "Real-time photoacoustic tomography of cortical hemodynamics in small animals," Journal of Biomedical Optics, 15(1), 010509-1-3 (2010).

[8] J. Xia, Z. Guo, K. Maslov et al., "Three-dimensional photoacoustic tomography based on the focal-line concept," Journal of Biomedical Optics, 16(9), 090505 (2011).

[9] M. Xu, and L. V. Wang, "Universal back-projection algorithm for photoacoustic computed tomography," Physical Review E, 71(1), 016706 (2005). 